\title{
Química Combinatória: moderna ferramenta para a obtenção de candidatos a protótipos de novos fármacos
}

\author{
Patrícia de Aguiar Amaral, Gilda Neves, Fabiane Farias, Vera Lucia Eifler-Lima*
}

Faculdade de Farmácia, Universidade Federal do Rio Grande do Sul

*Correspondência:

V. L. Eifler-Lima

Departamento de Produção de

Matéria-prima

Faculdade de Farmácia/UFRGS

Av. Ipiranga 2752, Rio Branco.

90610-000 - Porto Alegre, RS

eifler@farmacia.ufrgs.br
A Química Combinatória (QC) é atualmente uma das mais promissoras ferramentas para a descoberta e o desenvolvimento de novas moléculas com potencialidades terapêuticas. Nesta metodologia de síntese os produtos são formados simultaneamente e podem ser testados biologicamente em uma só vez, seja em forma de misturas ou separadamente. A principal meta desta nova metodologia é reduzir o tempo necessário para a obtenção de um novo fármaco. A sintese de quimiotecas pode ser realizada através da utilização de polímeros insolúveis, solúveis ou pela tradicional sintese em solução, sendo que cada uma delas apresenta características que podem ser vantajosas ou não, dependendo da metodologia utilizada. O desenvolvimento de técnicas de high throughput screening (HTS) devido às suas características peculiares necessita de tempo, razão pela qual a sintese de quimiotecas pequenas e racionais é enfatizada.
Unitermos

- Química Combinatória

- Síntese Orgânica em

Fase Sólida

- Planejamento Racional de Fármacos

- Química Medicinal

\section{INTRODUÇÃO}

Apesar do crescente desenvolvimento e dos evidentes avanços científico e tecnológico da indústria farmacêutica, existem ainda inúmeras doenças que necessitam de novos fármacos, com melhor perfil terapêutico para o seu tratamento, como por exemplo SIDA, Alzheimer, câncer, malária, entre outras (Wermuth, 1996). Assim, a busca por novos agentes terapêuticos ainda se faz necessária nos tempos de hoje.

Historicamente, a pesquisa para a descoberta de novas substâncias biologicamente ativas vem seguindo processo seqüencial, em que a síntese dos compostos ocorre de maneira artesanal, isto é, uma molécula de cada vez, escolhendo-as a partir de substâncias de origem natural ou sintética e, depois de exaustivas etapas de isolamento, purificação e caracterização, o candidato a protótipo de fármaco é submetido a testes para detecção de eventuais atividades farmacológicas (Gedeck, Willett, 2001). Este processo clássico é amplamente empregado em laboratórios de pesquisa de instituições de ensino e empresas farmacêuticas, mas apresenta como desvantagem o fato de ser relativamente lento e gerar reduzido número de derivados diferentes.

Atualmente, o planejamento racional de fármacos fundamenta-se basicamente no conhecimento da estrutura molecular dos receptores, o que possibilita elaborar substâncias com perfis farmacológicos mais definidos. Para tanto, as hipóteses são elaboradas fundamentando-se no conhecimento das propriedades físico-químicas dos compostos ativos e seus respectivos sítios moleculares de ação (Folkers, Kubinyi, 1997). Entretanto, como muitas vezes a estrutura dos receptores não é conhecida admite-se que a gênese de moléculas potencialmente bioativas seja realizada sem levar em conta sua natureza (Villar, Koehler, 2000). 
O progresso mais recente consiste na construção de uma só vez de coleções de substâncias que servirão para estudos de relação estrutura/atividade (REA), ou seja, para descobrir uma nova entidade química candidata a protótipo de fármaco (Gordon, Kerwin, 1998; Fenniri, 2000). É nesta etapa que a química combinatória torna-se uma ferramenta importante no progresso da química medicinal. Atualmente, grande variedade de novas moléculas vem sendo sintetizada através da Química Combinatória (QC), intensiva área de pesquisa desde o seu surgimento no início dos anos 90 (Brocchini, 2001). Esta estratégia tomou impulso no momento em que foi incorporada à química medicinal, a partir dos trabalhos de Elmann com a síntese de benzodiazepinas (Bunin, Ellman, 1992), e de DeWitt na síntese de quimiotecas de hidantoínas (DeWitt et al., 1993). Ela permite utilizar a síntese automatizada e miniaturizada e a farmacologia de forma interativa, reduzindo consideravelmente o tempo da pesquisa, uma vez que cria rapidamente uma coleção de substâncias análogas, com diferentes grupos farmacofóricos ou conformações, objetivando acelerar a descoberta de potenciais candidatos a fármacos (Gordon, Kerwin, 1998; Fenniri, 2000).

\section{QUIMIOTECAS}

Em QC, uma quimioteca é uma coleção de moléculas sintetizadas simultaneamente em forma de misturas ou isoladas, a partir de um ou mais substratos de partida. As quimiotecas podem ser constituídas de dezenas, centenas ou milhares de estruturas diferentes. Historicamente, as primeiras quimiotecas sintetizadas foram as de peptídeos (Merrifield, 1963). Contudo, devido aos problemas de biodisponibilidade apresentados por estas estruturas, aliados à sua baixa diversidade química, as investigações voltaram-se para a síntese de moléculas orgânicas mais simples, de natureza não polimérica (Kubinyi, 1995). De fato, este interesse dirige-se cada vez mais à criação de quimiotecas de heterociclos substituídos devido à grande relevância terapêutica de derivados apresentando este padrão de funcionalização (Franzén, 2000). A experiência acumulada que havia para sintetizar peptídeos contribuiu para a aplicação e o desenvolvimento desta metodogologia.

Atualmente, a QC utiliza-se do planejamento racional para a criação de suas quimiotecas, nas quais a síntese simultânea de vários compostos por coleção aumenta a probabilidade de se chegar a uma substância protótipo. Adicionalmente, o emprego de recursos computacionais modernos e da modelagem molecular, que aliam as propriedades estruturais ou físico-químicas dessas moléculas, permitem o planejamento racional da diversidade (Villar, Koehler, 2000).
Quimiotecas pequenas são mais facilmente caracterizadas e possuem alta reprodutibilidade química, sendo adequadas para o aperfeiçoamento de propriedades de um protótipo e estudos de relação estrutura-atividade (REA). Grandes quimiotecas são mais indicadas quando o objetivo é identificar o protótipo. Desta forma, a QC pode ser empregada tanto para descobrir novas moléculas potencialmente bioativas quanto para otimizar sua atividade e/ou diminuir sua toxicidade, estando por trás disso um intenso planejamento de todas as etapas do processo (da síntese à avaliação farmacológica) e a escolha correta da estratégia de síntese a ser utilizada (Brocchini, 2001).

Quimiotecas podem ser diferenciadas de acordo com o modo de síntese escolhido e a estratégia sintética empregada (Figura 1). Elas podem ser preparadas através de síntese em solução ou então com o auxílio de um polímero insolúvel, originando o que se conhece hoje como a Síntese Orgânica em Fase Sólida - SOFS (Marquardt, Eifler-Lima, 2001). Já quanto à estratégia, podem-se construir quimiotecas através da síntese em paralelo, originando grande quantidade de compostos já isolados, ou através da síntese de misturas.

A possibilidade de formação das quimiotecas na forma de misturas de substâncias ocorre, por exemplo, quando à uma molécula inicial (building block) se adicionada mistura de reagentes resultando na formação de mistura de compostos (Dias, Corrêa, 2001). Esta estratégia de síntese acarreta a realização dos testes biológicos com as misturas, de modo que, no caso de uma das quimiotecas apresentar uma resposta interessante, o próximo passo a seguir é a sua ressíntese (Thompson, Ellman, 1996; Marquardt, Eifler-Lima, 2002). A preparação de quimiotecas em solução, na forma de grandes misturas ( $c a$ 1000 substâncias), diminui da mesma maneira o tempo de síntese, mas conduz a algumas dificuldades adicionais (Thompson, Ellman, 1996). As cinéticas distintas existentes em cada reação química podem levar à formação de produtos em diferentes concentrações, sendo, então, a síntese de quimiotecas menores o procedimento mais indicado e adotado atualmente por muitos pesquisadores (Fenniri, 2000).

\section{MODOS DE SÍNTESE DAS QUIMIOTECAS}

\section{Síntese orgânica em fase sólida - SOFS}

A síntese orgânica em fase sólida (SOFS) é sem dúvida a maneira mais utilizada para a obtenção de quimiotecas combinatórias. Este tipo de síntese emprega suportes sólidos, constituídos por polímeros insolúveis, também conhecidos como resinas, que possuem uma 


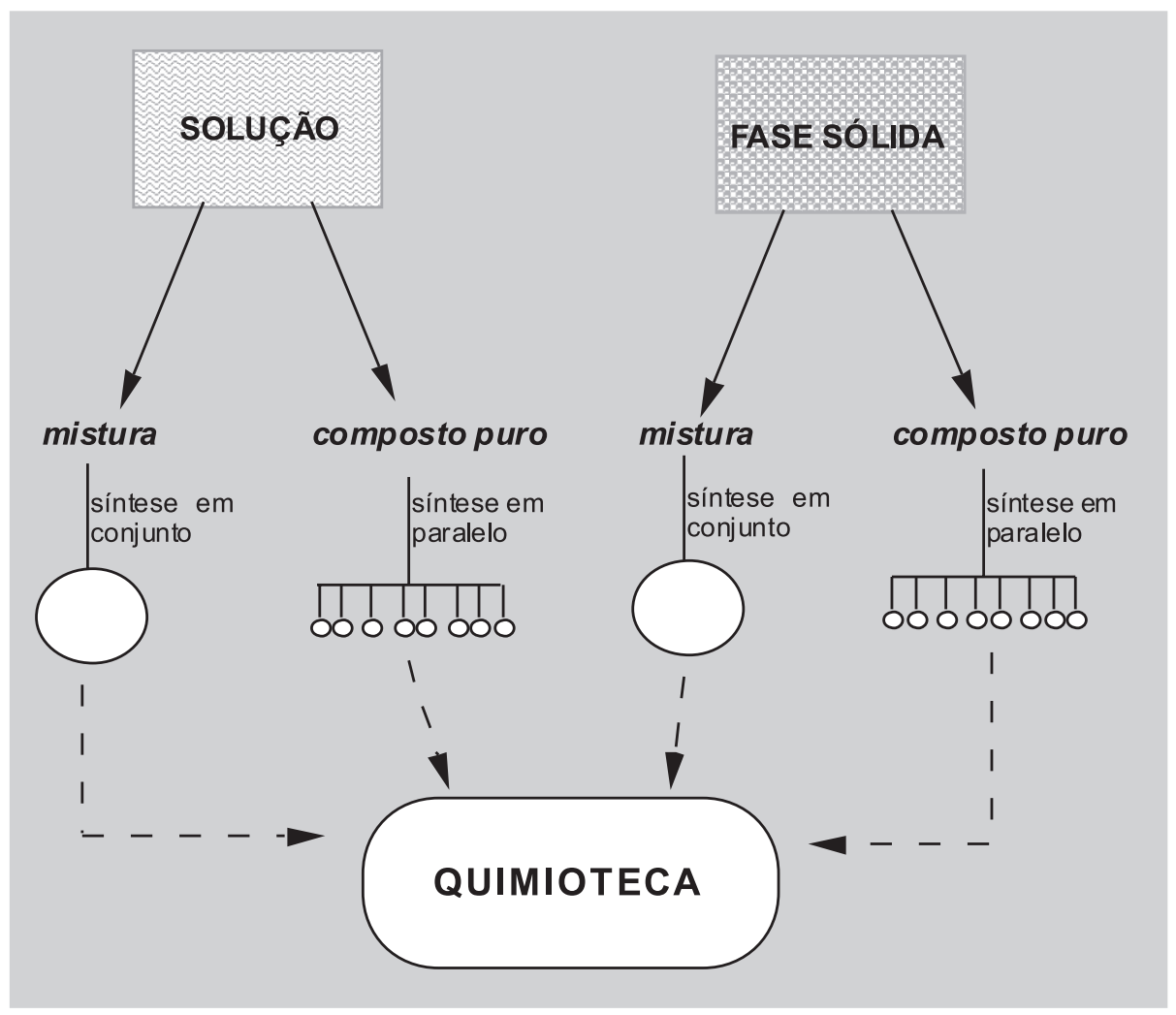

FIGURA 1 - Estratégias sintéticas empregadas para a construção de quimiotecas.

subunidade responsável pela sustentação física da molécula (estrutura polimérica inerte) e outra parte, conhecida como ligante (linker), responsável pelo acoplamento ao material de partida. Após as transformações químicas realizadas em outra parte da molécula, as resinas são separadas do produto por meio de algumas estratégias de clivagem (Marquardt, Eifler-Lima, 2001).

O primeiro relato sobre o emprego da SOFS data de 1963, quando Bruce Merrifield desenvolveu metodologia para a síntese de peptídeos, utilizando polímeros insolúveis e inertes, que serviram de suportes para a síntese (Merrifield, 1963). Esta técnica apresentou vantagens na quimiosseletividade das reações e, conseqüentemente, diminuição de reações paralelas. Entretanto, a maior vantagem da SOFS é a simplificação da etapa de purificação dos produtos sintetizados, que passa a consistir de simples processo de lavagem e filtração em cada etapa da rota sintética. Dessa maneira, eliminam-se excesso de solventes, reagentes e possíveis produtos indesejados não covalentemente ligados à resina. A última etapa da rota sintética consiste na clivagem da molécula do polímero para obtenção do produto final com elevado grau de pureza (Marquardt, Eifler-Lima, 2001). O pioneirismo dos trabalhos de Merrifield conduziu-o ao prêmio Nobel de Química no ano de 1984 com a síntese da bradicinina (Dulac et al., 2001). Na década de 1980, esses trabalhos foram retomados abrindo perspectivas para o desenvolvimento de outros polímeros com diferentes ligantes e estruturas diferenciadas da resina Merrifield (Blackburn et al., 1997; Rano et al., 2000). Atualmente, esta metodologia é rotineiramente empregada em laboratórios europeus e norteamericanos, acadêmicos ou de empresas farmacêuticas.

Para ilustrar a relevância do uso de suportes sólidos na pesquisa pela busca de novos fármacos, podemos citar como exemplo um método versátil para a síntese de arilalcanolaminas diversamente substituídas empregando carbamatos imobilizados (Figura 2). A metodologia foi empregada com sucesso na síntese de ariletanolaminas e arilpropanolaminas com altos rendimentos e pureza dos produtos (Srinivasan et al., 2001).

\section{O desafio do monitoramento}

Diferentemente da síntese em solução, que faz uso da cromatografia e/ou da ressonância magnética nuclear, a síntese em fase sólida possui a etapa de acompanhamento das reações como um fator limitante. Esta deficiência acarreta imprecisão dos tempos reacionais e incertezas nos rendimentos. Por isso, inúmeros estudos visam ao desenvolvimento de técnicas para o acompanhamento das reações cujos produtos a serem monitorados estão acoplados 


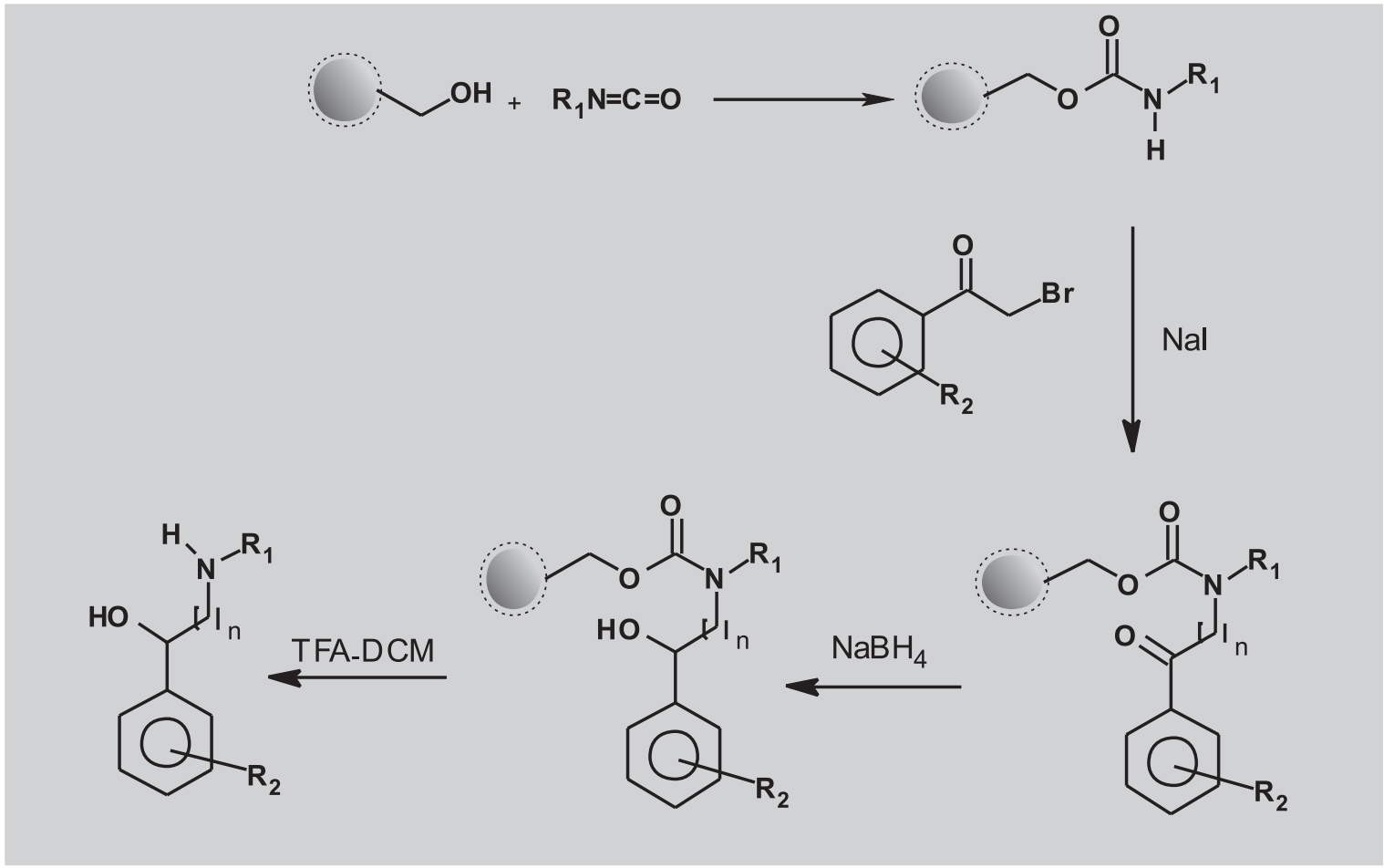

FIGURA 2 - Rota sintética, empregando a SOFS, para a síntese de arilpropanolaminas.

às resinas. Essas investigações sugerem o uso da espectroscopia no infravermelho (IV) para o monitorização de reações em fase sólida (Yan, 1998; de Miguel, Shearer, 2001), além de outras técnicas como: eletroforese capilar, espectrometria de massas, ressonância magnética nuclear e cromatografia líquida acoplada à espectrometria de massas (Fauchére et al., 1998).

A espectroscopia na região do infravermelho com o uso de microscópio de alta resolução é um dos métodos analíticos mais estudados com este objetivo, possuindo utilidade na determinação do tempo final de reação com maior segurança e, conseqüentemente, otimização do processo como um todo (Yan, 1998; Yan et al., 1999; Pivonka, 2000). Entretanto, ainda não pode ser metodologia de rotina, principalmente devido ao seu alto custo. Para tanto, sugere-se o uso do IV com pastilhas de $\mathrm{KBr}$ e correção da linha de base do espectro. O simples emprego desta medida operacional no espectrômetro fornece espectros com alta qualidade e definição, podendo ser empregado rotineiramente para o controle das reações em fase sólida (EiflerLima et al., 2002). O método consiste basicamente em observar o aparecimento e/ou o desaparecimento de bandas características aos intermediários formados. Para isso escolhe-se uma banda representativa da resina como padrão interno, como por exemplo a banda de absorção em 1450 $\mathrm{cm}^{-1}$ pertencente à ligação $\mathrm{C}=\mathrm{C}$ aromática da resina Merrifield. Desta maneira o uso da espectroscopia no IV com pastilhas de $\mathrm{KBr}$ para monitorar reações em fase sólida pode ser explorado, o que é facilitado pela existência deste aparelho na maioria dos laboratórios de química orgânica das universidades.

\section{Síntese em solução}

A síntese de quimiotecas em solução consiste na dissolução de todos os reagentes no solvente. Os pesquisadores que se dedicam a esse tipo de estratégia sintética preconizam diversas vantagens destas, quando comparadas à SOFS, como veremos a seguir. Uma das vantagens preconizadas para esse tipo de síntese na obtenção de quimiotecas está justamente no maior contato que ocorre entre os reagentes, aumentando a probabilidade de deslocamento da reação para a obtenção do produto desejado (Gayo, 1998), embora não existam até o presente trabalhos de cinética química conclusivos que demonstrem que as reações em solução são mais rápidas que as realizadas por SOFS. Outro fator considerado é o custo, pois não requer excesso de reagentes como ocorre na SOFS. Como não existem as etapas de acoplamento à resina, nem de clivagem, o tempo de síntese é menor, fornecendo o produto solúvel, diretamente no meio reacional. Além disso, existe enorme repertório de reações químicas desenvolvidas em solução, facilitando os caminhos de obtenção dos produtos, fazendo com que esta metodologia seja mais 
adequada para a otimização do protótipo (Gayo, 1998). Pode ser aplicada tanto para quimiotecas de produtos individuais quanto de misturas de produtos.

Contudo, algumas desvantagens para a aplicação desta metodologia em química combinatória podem ser evidenciadas. A principal desvantagem da preparação de coleções combinatórias em solução, com grau de pureza confiável para a posterior avaliação farmacológica, consiste na purificação dos intermediários e produtos, que é particularmente difícil, demandando período de tempo adicional (Gayo, 1998; Corrêa, Dias, 2001). Estas reações são limitadas a seqüências curtas na rota sintética, evitando procedimentos sintéticos com muitas etapas. Reações que requeiram excesso de material de partida ou de reagentes não voláteis também são evitados, pois produzem importantes impurezas ao final da síntese. Adicionalmente, na síntese em solução é imperativo que ocorra a completa conversão dos reagentes a produtos com pouca ou nenhuma formação de subprodutos. Como pode ser observado, a combinação de todos estes fatores limita a aplicação da síntese em solução na QC.

Para contornar esses problemas alguns pesquisadores têm desenvolvido técnicas a fim de viabilizar a aplicação da síntese em solução na QC. Dentre estes pode-se citar Curran e colaboradores (2000) e Studer e colaboradores (1997), que desenvolveram técnica de extração líquido-líquido, que consiste na utilização de suportes fluorados, também chamada de "síntese combinatória em fase fluorosa". Nesta síntese utilizam-se solventes perfluorcarbonados (PFC), que são imiscíveis tanto em água quanto na maioria dos solventes orgânicos, constituindo-se em uma "terceira fase", facilmente extraída (Figura 3 ).

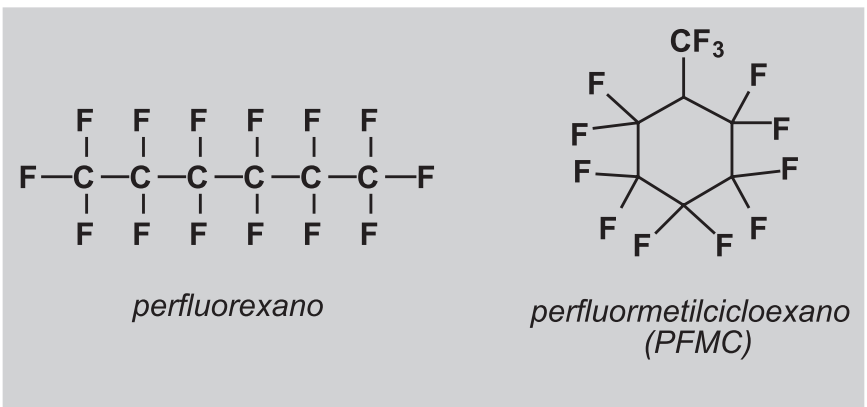

FIGURA 3 - Dois exemplos de solventes perfluorados.

Na síntese em fase fluorosa, o substrato é ligado temporariamente ao reagente fluorado, que pode ser liberado em qualquer etapa da síntese. A separação ocorre com a partição em fases diferentes com base na presença ou ausência do reagente fluorado, com uma extração líquido/líquido, que pode ser em duas fases (flúor-orgânica ou flúor-água) ou três fases (flúor-orgânica-água) (Curran, 2000).

A aplicação de polímeros como "removedores de impurezas" (material de partida em excesso ou subprodutos de reações paralelas), conhecidos como scavengers, também vem sendo metodologia cujo uso está em crescente ascensão pelos praticantes de síntese orgânica em solução (Booth, Hodges, 1997). Isto porque estes polímeros reagem com os grupos funcionais das impurezas formando ligação covalente, sendo retirados da mistura reacional pelo simples processo de filtração (Figura 4). Os scavengers possuem no seu linker grupamentos de caráter nucleofílico ou eletrofílico como isocianatos, aldeídos, aminas, entre outros. Este tipo de resina, em sua maioria derivadas da resina Merrifield, diminui de forma considerável os contaminantes usuais, que dificultam a separação e identificação das quimiotecas sintetizadas em solução (Gayo, 1998).

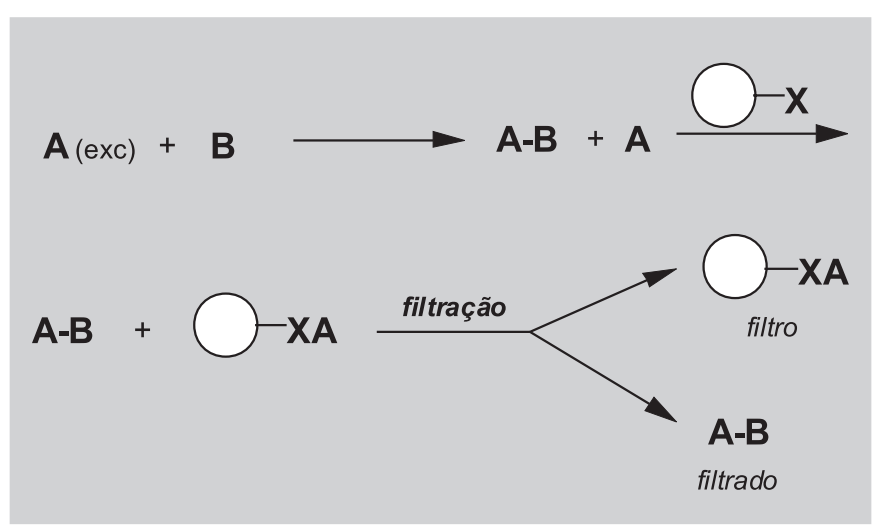

FIGURA 4 - O substrato A reage com B formando o produto $\mathrm{AB}$ e $\mathrm{A}$ excedente, que reage com a resina e é eliminado por filtração fornecendo o produto $\mathrm{AB}$. $\mathrm{O}$ resina; $\mathrm{X}$ - grupo funcional.

\section{Desviando as dificuldades: polímeros solúveis}

Outro método emergente visando à utilização de síntese em solução para gerar quimiotecas preconiza o emprego de suportes poliméricos solúveis como auxiliares na síntese (Borman, 2001a,b). Este tipo de reação foi descrito pioneiramente por Janda e colaboradores, que desenvolveram homopolímeros lineares solúveis, nos quais a reação é realizada em meio completamente homogêneo (Gravert, Janda, 1996). Esta estratégia tem por objetivo combinar as vantagens de ambas as tecnologias: a SOFS e a síntese em solução, chamada de "síntese orgânica em fase líquida SOFL". Nesta metodologia o polímero solubilizado no meio reacional é precipitado através da adição de determinados solventes, como éter etílico ou óxido de nitrila em éter etílico, seguindo-se, então, de filltração e lavagem, o que 
leva ao isolamento dos produtos de interesse. Este tipo de síntese permite o acompanhamento da reação através das técnicas clássicas, como a cromatografia em camada delgada (CCD), além das demais vantagens mencionadas para a síntese em solução.

Como exemplo de sua utilização, polímeros solúveis foram empregados na síntese de $\beta$-aminoálcoois, levando à obtenção do propranolol em bons rendimentos $\mathrm{e}$ alto grau de pureza (Figura 5) (Hans et al., 1995).

Contudo, a escolha por este tipo de técnica ainda acarreta algumas desvantagens de natureza experimental e as dificuldades decorrentes de sua utilização muitas vezes ultrapassam os benefícios obtidos. A principal desvantagem deste último tipo de polímero é que as reações somente podem ser efetuadas em solventes nos quais ele é solúvel, limitando seu uso. Outra características destas resinas é que elas podem formar no meio reacional massa altamente viscosa ("grudenta") que torna a filtração muito difícil. Por outro lado, estas resinas permitem o monitoramento por espectroscopia de $\mathrm{RMN}{ }^{1} \mathrm{H} \mathrm{e}{ }^{13} \mathrm{C}$ de alta resolução (Sherrington, 1998).

\section{ESTRATÉGIAS A PARA SÍNTESE DE QUIMIOTECAS}

Sem dúvida alguma, a tendência atual da química combinatória é o desenvolvimento de métodos de síntese em fase sólida de quimiotecas tanto de substâncias isoladas (em paralelo e em "beads") quanto em misturas, pela técnica de "misturar e dividir" (pool-and-split synthesis), como será comentado a seguir.

\section{Síntese em paralelo - substâncias isoladas}

A síntese combinatória em paralelo consiste na sín- tese simultânea de série de compostos em distintos meios de reação. As etapas são realizadas simultaneamente, em compartimentos individuais, geralmente em placas com 96 pocinhos, nas quais cada pocinho é um diferente meio reacional e em cada um destes meios é sintetizado um único e definido produto, o qual pode ser prontamente identificado. Atualmente, existem placas com maior número de pocinhos (384 e 1536) e a escolha entre uma destas dependerá da diversidade da síntese e do número de compostos a serem sintetizados (Dias, Corrêa, 2001; Wolcke, Ullmann, 2001; Marquardt, Eifler-Lima, 2002).

A síntese em paralelo é sempre planejada segundo orientação pré-estabelecida, não se tratando simplesmente da realização de múltiplas reações com formação de produtos que guardam pouca ou nenhuma relação estrutural entre si. As quimiotecas obtidas formam uma "família" na qual ocorrem poucas e definidas variações estruturais e conformacionais entre seus componentes (Marquardt, Eifler-Lima, 2002). Esta estratégia de síntese pode ser realizada tanto em solução como através do emprego de suportes sólidos. Entretanto, devido às vantagens da metodologia mencionada anteriormente, acredita-se que a realização da síntese paralela em fase sólida seja a estratégia mais vantajosa.

\section{Split-and-pool synthesis - síntese de misturas}

A formação de pequenas quimiotecas na forma de misturas de substâncias ocorre quando à molécula inicial se adiciona, num mesmo recipiente, a mistura de reagentes da mesma classe química. Após o período de tempo necessário haverá a formação de mistura de produtos congêneres (Dias, Corrêa, 2001). O emprego desta estratégia induz à realização dos testes biológicos diretamente com as misturas. Caso umas das quimiotecas apresente uma resposta

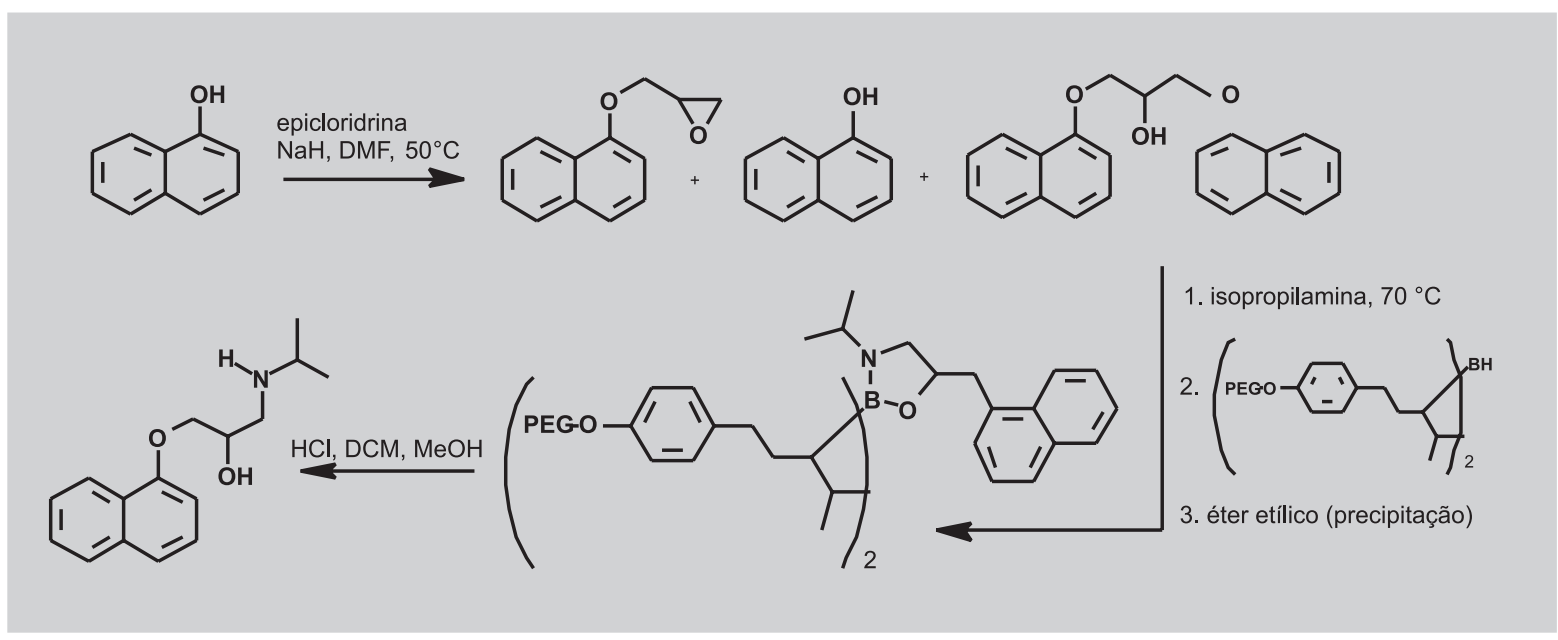

FIGURA 5 - Síntese de $\beta$-aminoálcoois: obtenção do propranolol utilizando um polímero solúvel. 
interessante, avança-se para o passo seguinte, que seria a ressíntese desta quimioteca, através de estratégias de dissociação (deconvolution) (Figura 6), para descobrir a molécula responsável pela atividade observada (Marquardt,

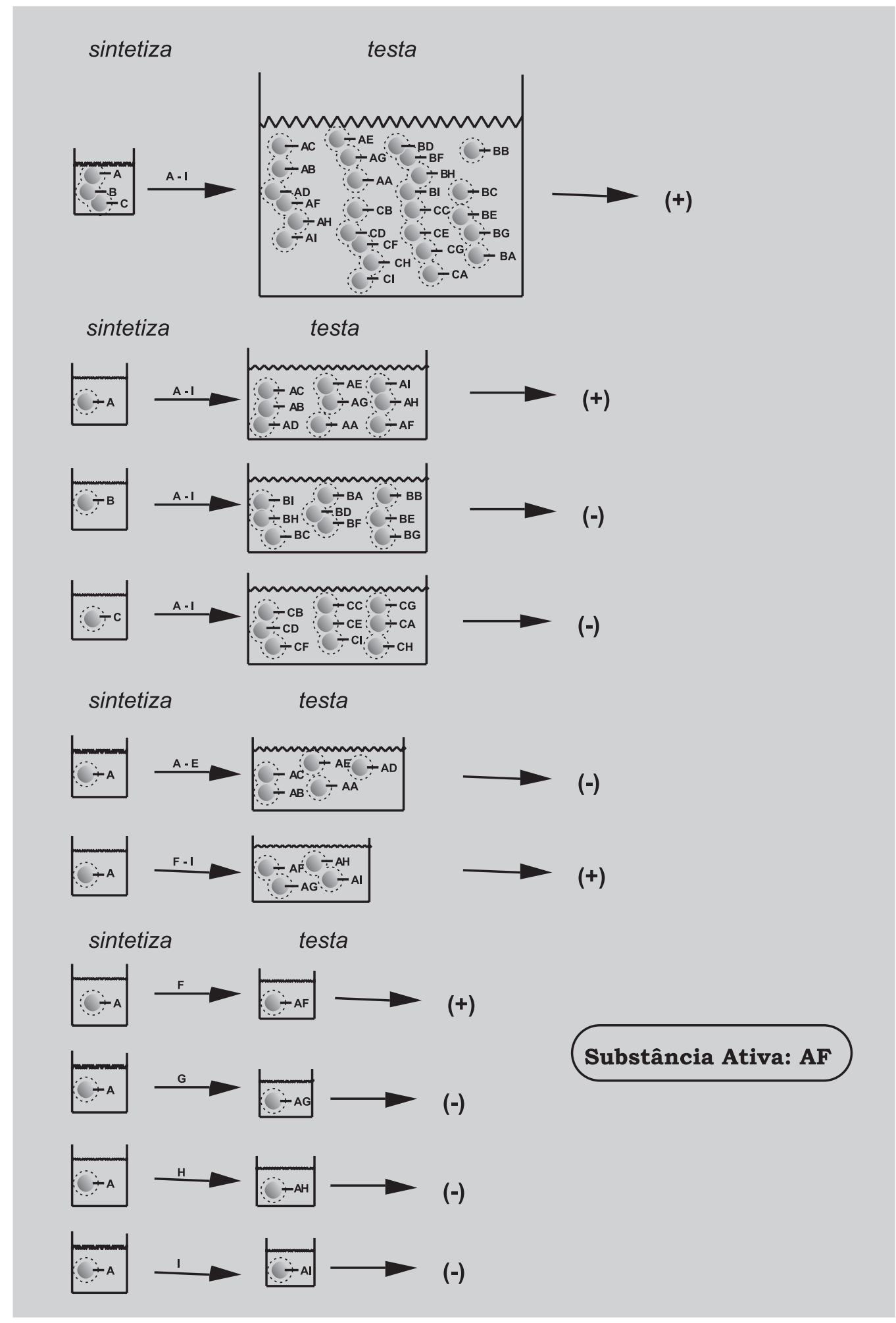

FIGURA 6 - Síntese de misturas. A mistura é sintetizada e testada e o resultado é positivo. Para chegar ao composto responsável pela atividade, procede-se à dissociação da mistura com ressínteses e repetição dos testes com quimiotecas cada vez menores. 
Eifler-Lima, 2002). Quimiotecas de misturas podem ser feitas em fase sólida ou em solução e é uma estratégia eficiente para a identificação do protótipo. Para um estudo de relação estrutura/atividade (REA) quimiotecas de compostos puros são as ideais (Boger, Goldberg, 2000).

Para a formação de quimiotecas maiores ( ca 1000 10.000 moléculas) prefere-se a síntese de misturas conhecida como split-and-pool synthesis. Sua concepção básica consiste em grupos de compostos, os quais são modificados quimicamente, divididos, reagrupados e modificados novamente em uma série de passos seqüenciais. Sucessivamente, formam-se produtos em progressão geométrica a cada nova etapa de separação e mistura (Figura 7).

A vantagem do uso desta metodologia consiste na ampla diversidade química gerada, com o uso de muitos building blocks diferentes, fazendo com que seja a mais difundida das metodologias empregadas em QC, inclusive utilizada como exemplo ilustrativo quando se aborda o assunto.

\section{ESTRATÉGIAS UTILIZADAS NA AVALIAÇÃO FARMACOLÓGICA DE QUIMIOTECAS COMBINATÓRIAS}

Antes de abordarmos as estratégias mais empregadas para a avaliação biológica e identificação de qual composto apresentou melhor resposta biológica, é necessário mencionar que o sucesso desse processo está inteiramente ligado à sensibilidade e especificidade do ensaio e da técnica de screening. O candidato mais potente talvez não venha a ser descoberto no primeiro screening. Pode ocorrer que um ligante com baixa afinidade venha a ser detectado e, num segundo momento, usado como protótipo na ressíntese de quimiotecas menores. O screening pode ser realizado com o produto ainda ligado à resina ou então solubilizado (descartada a resina), isoladamente ou em misturas do tipo split-and-pool. Isto define o tipo de ensaio a ser usado e, ultimamente, o tamanho das quimiotecas a serem ensaiadas (Fenniri, 2000).

Concomitantemente com o aperfeiçoamento das técnicas de química combinatória, farmacólogos e biólogos vêm desenvolvendo os chamados ensaios biológicos automatizados (high throughput screening - HTS), um processo rápido de determinação da atividade de amostras de uma quimioteca combinatória, geralmente de forma paralela em placas com 96 poços acoplados a leitores de emissão de fluorescência, com o objetivo de descobrir protótipos biologicamente ativos (Fenniri, 2000; Dias, Corrêa, 2001).

Além das placas, outras técnicas utilizadas nos ensaios de quimiotecas são: permeação em gel (bastante similar às técnicas de avaliação de atividade antimicrobiana) e seleção por afinidade, a qual constitui-se na determinação da afinidade de ligação do composto por determinada macromolécula, sem avaliação de qualquer atividade (Sigal, Chelsky, 1998). A maioria dos alvos biológicos utilizados é constituída de variações de quatro enfoques básicos: ensaios enzimáticos, cultura de células íntegras, ensaios de binding e de ligação a alvos específicos altamente purificados sem predição de atividade (Beutel, 1998).

A escolha da técnica e do tipo de ensaio a serem utilizados na avaliação farmacológica das quimiotecas sintetizadas está estreitamente relacionada ao alvo terapêutico selecionado. Contudo, a estratégia e o modo de síntese utilizados devem ser igualmente considerados. Quimiotecas originadas da síntese em paralelo podem ser facilmente avaliadas em placas do mesmo tamanho que as já utilizadas na síntese, sendo o composto ativo facilmente identificado, devido à presença de um único composto por pocinho (Beutel, 1998; Sigal, Chelsky, 1998). Já as quimiotecas de misturas podem ser ensaiadas sem o isolamento dos compostos sintetizados. Este ensaio ocorre de forma muito similar à estratégia do fracionamento bioguiado de extratos vegetais: neste a mistura é testada e, apresentando atividade promissora, é retornada ao químico para que seja identificada e ressintetizada ("fracionada") e, assim, sucessivamente, até o isolamento de um ou mais produtos promissores como está esquematizado na Figura 6. Porém, esta estratégia possui como principal desvantagem a provável interação entre os compostos presentes na quimioteca, que podem possuir efeitos sinérgicos ou antagônicos, dificultando a interpretação dos resultados obtidos.

Há, ainda, a possibilidade de, com quimiotecas originadas de um procedimento de síntese em fase sólida, serem realizados ensaios com os produtos ainda ligados à resina, também denominados ensaios do tipo on-bead. Esta estratégia agiliza a identificação de substâncias ativas, uma vez que elimina a etapa de clivagem dos produtos da resina, bem como etapas de purificação dos mesmos. Ao utilizar uma estratégia de síntese na qual cada bead contém apenas um produto, a identificação e isolamento do composto ativo fica muito facilitada, consistindo apenas na separação do bead ativo dos demais (Beutel, 1998; Sigal, Chelsky, 1998).

Justamente a presença da resina acarreta certas limitações para este tipo de ensaio. Primeiramente, todos os reagentes devem encontrar-se em solução para garantir sua difusão ao interior da resina, impossibilitando a utilização de certos ensaios em células íntegras, devido ao pouco acesso dos produtos aos alvos intracelulares. O composto sintetizado, quando ligado à resina, pode encontrar-se em uma 


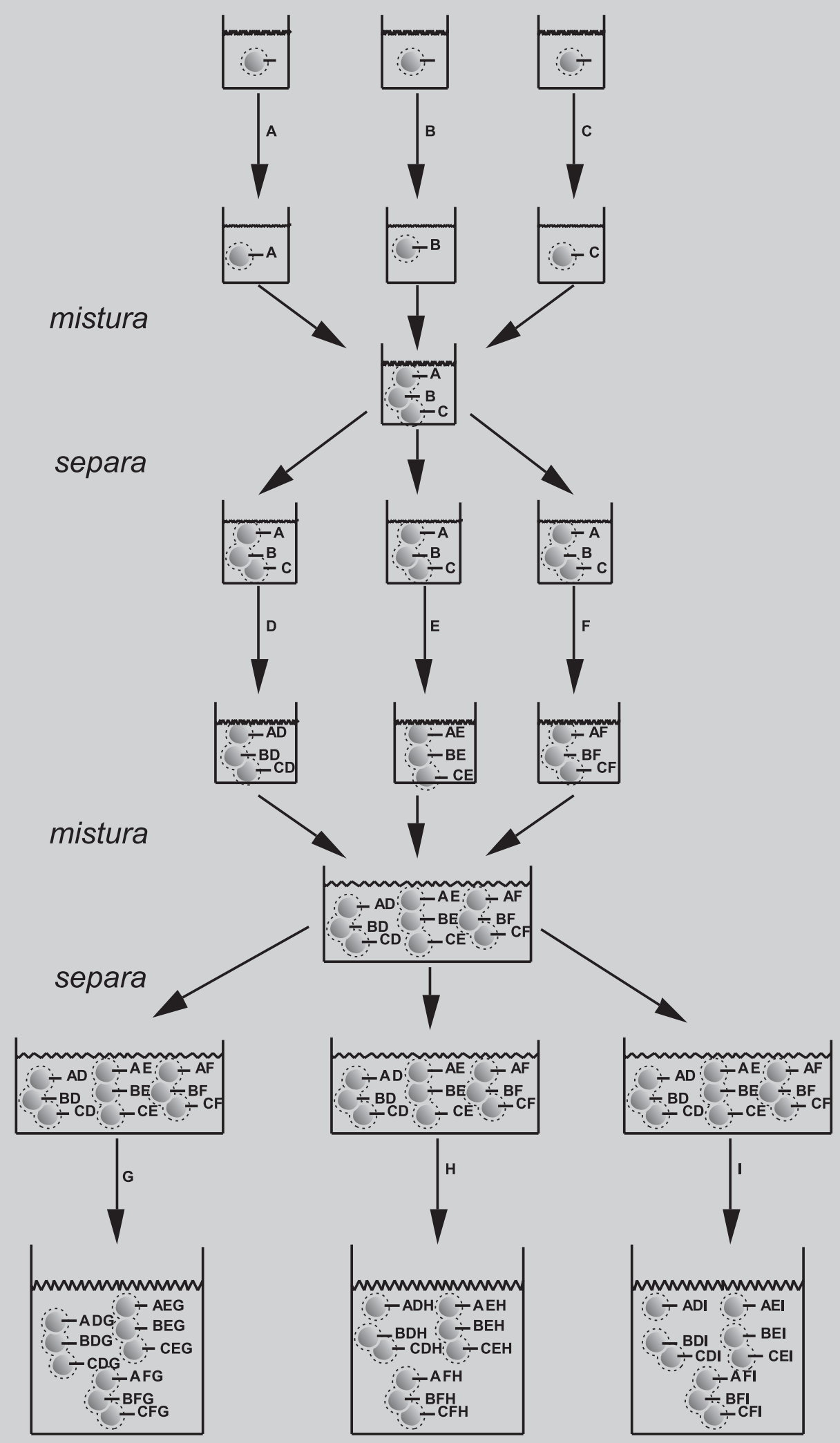

FIGURA 7 - Split-and-pool synthesis. 
conformação diferente de sua conformação em solução devido, principalmente, à natureza hidrofóbica do polímero, o que pode causar interferência na interação da molécula com o alvo biológico. Apesar destes obstáculos, a utilização de ensaios on-bead é de grande valia principalmente na préseleção de um grupo de compostos para posterior avaliação em solução e confirmação de sua potencial atividade (Sigal, Chelsky, 1998).

Uma das formas de contornar estas limitações é a realização de determinadas técnicas de permeação em gel, nos quais os beads são distribuídos em uma fina camada de gel e apenas parte do produto é clivado da resina. Esta parcela de substrato clivado é que vai, então, se difundir no gel e atingir o alvo biológico, com a conseqüente formação de um halo de fluorescência em torno do bead de origem. Este pode, então, ser isolado com parte do produto ainda ligado para posterior clivagem e identificação estrutural. Estas características fazem com que este tipo de ensaio agregue a maior confiabilidade dos ensaios em solução e a facilidade de identificação do composto ativo dos ensaios on-bead (Beutel, 1998).

A constante necessidade de reduzir o tempo de descoberta de um novo fármaco, bem como de lidar com a grande quantidade de compostos sintetizados através das diversas estratégias da química combinatória frente a gama de novos alvos advindos dos estudos do genoma humano, têm levado à uma completa reavaliação das tecnologias utilizadas nos ensaios farmacológicos in vitro. Neste contexto, uma das estratégias a ser utilizada é a miniaturização, na qual é necessário o estabelecimento de condições ótimas de ensaio para uma escala de volume de microlitros, agilizando todo o processo de teste de novos compostos sem aumento dos custos (Wolcke, Ullmann, 2001).

Um ensaio é considerado miniaturizado quando o volume final do meio de incubação não ultrapassa $5 \mu \mathrm{L}$, permitindo à utilização de placas com 1536 pocinhos ou mais. Esta estratégia resulta em economia de solventes e reagentes de, no mínimo, 20 vezes em relação ao formato tradicional de 96 pocinhos (Wolcke, Ullmann, 2001).

Teoricamente, qualquer técnica já utilizada rotineiramente no formato de 96 pocinhos pode ser miniaturizada. Porém, na prática surgem algumas complicações, sendo uma das mais importantes o limite de detecção das técnicas de identificação, baseadas principalmente na emissão de fluorescência total (Sigal, Chelsky, 1998). Os pequenos volumes utilizados nos ensaios levam à redução na superfície de contato entre os compostos e o alvo terapêutico e à necessidade de fina automação do sistema de pipetagem, bem como de proteção contra a evaporação das pequenas quantidades de reagentes, garantindo maior uniformidade em todos os pocinhos (Wolcke, Ullmann,
2001). Outra característica importante é que os ensaios para HTS possibilitem a utilização de solventes orgânicos, objetivando sobretudo a solubilização de substâncias hidrofóbicas.

\section{CONSIDERAÇÕES FINAIS}

A QC vem se constituindo em importante ferramenta no planejamento racional de novos fármacos, despertando o interesse das empresas farmacêuticas. Esse interesse é devido, em parte, à missão primordial da QC: a busca por maior diversidade molecular no mais curto espaço de tempo possível. Acredita-se que a modelagem molecular, que é capaz de prever qual o tipo de ligante específico para uma determinada biomolécula (bio-receptor), juntamente com a QC, com seus robôs miniaturizados, acelerem o processo de descoberta de novos fármacos (Watkins, 2002).

O surgimento da QC no começo da década de 90 com a proposta de obtenção de moléculas bioativas em menor espaço de tempo e, conseqüentemente, a probabilidade maior de se chegar rapidamente a um novo fármaco, levou a indústria farmacêutica a investir de maneira significativa nesta área. Como exemplos têm-se a aquisição e o desenvolvimento de equipamentos adequados e pessoal altamente qualificado, evidentemente visando ao rápido retorno financeiro com a possibilidade de novas patentes (Caldwell, 1998).

Atualmente, sabe-se que a síntese de milhares de moléculas torna difícil tanto a identificação da substância ativa quanto os ensaios farmacológicos. Dessa maneira propõe-se a síntese de quimiotecas menores, visando à maior potência e seletividade da sua atividade biológica ou até mesmo a presença de outra atividade, através do planejamento elegante, que inclui a modelagem molecular, uma rota sintética muito bem delineada e o tipo de ensaio biológico a ser desenvolvido. Soma-se a isso a possibilidade de desenvolver estudos de REA e de quantificação de relação estrutura atividade (QSAR) com essas quimiotecas.

Para a realização dos testes farmacológicos as quimiotecas são testadas em ensaios biológicos automatizados, possibilitando, dessa forma, a avaliação in vitro de grande número de compostos. Entretanto, o desenvolvimento de técnicas de HTS, devido às suas características peculiares, necessita de tempo e não atende, ainda, à demanda de quimiotecas de milhares de compostos, razão pela qual a síntese de quimiotecas pequenas e racionais é novamente enfatizada.

Como pode ser observado, existe ainda muito trabalho relacionado à rotina a ser desenvolvido e otimizado, com novos conceitos a ser introduzidos e explorados no campo da QC. É inegável que esta moderna metodologia 
promoveu um grande impacto na química medicinal, embora a maior parte das aplicações industriais ainda não constem da literatura e existam ainda muitos problemas no que diz respeito às patentes (Caldwell, 1998). Entretanto, podese esperar que haja aumento da eficiência na geração de quimiotecas mais específicas e o desenvolvimento dos métodos analíticos de identificação. Neste sentido, é importante evidenciar que o tempo que se "perde" na otimização do processo, se ganha depois, na diversificação (Huesgo, 2001). Visto que o número de artigos publicados tem aumentado significativamente a cada ano, a expectativa para o futuro é de maior disseminação da QC no meio acadêmico brasileiro e, quem sabe, no setor produtivo nacional.

\section{ABSTRACT}

\section{Combinatorial chemistry: advanced tool for drug leads discovery}

Nowadays, Combinatorial Chemistry is one of the most promising tools used in the design and discovery of new molecules potentially useful in the therapeutics. In this methodology, the reaction products are synthesized simultaneously and can be evaluated once, either in a complex mixture or as isolated compounds. The main aim of this new approach is the time for the development of new drugs. The synthesis of combinatorial libraries can be performed using either insoluble or soluble polymers and also by the traditional organic synthesis in solution. Each one of these approaches presents features that may be advantageous depending on the final objective of the synthetic process. The development of high throughput screening (HTS) assays takes much time, which makes the synthesis of small and well designed combinatorial libraries the most recommended strategy.

UNITERMS: Combinatorial Chemistry. Solid Phase Organic Synthesis. Rational Drug Design. Medicinal Chemistry

\section{AGRADECIMENTOS}

Ao CNPq e à CAPES, pela concessão de bolsa de Mestrado das alunas P. A., F. F. e G. N.

\section{REFERÊNCIAS BIBLIOGRÁFICAS}

BEUTEL, B. A. Strategies for screening combinatorial libraries. In: GORDON, E.M.; KERWIN JR., J.F., ed. Combinatorial chemistry and molecular diversity in drug discovery. New York: Wiley-Liss, 1998. p. 421-432.
BLACKBURN, C.; ALBERICIO, F.; KATES, S. A. Functionalized resins and linkers for solid-phase synthesis of small molecules. Drugs Fut., Barcelona, v. 22 , n. 9 , p. $1007-1025,1997$.

BOGER, D.L.; GOLDBERG, J. Mutli-step solution phase combinatorial synthesis. In: FENNIRI, H. ed. Combinatorial chemistry. Practical approach. Oxford: Oxford University Press, 2000. cap. 10, p. 303-326.

BOOTH, R. J.; HODGES, J. C. Polymer-support quenching reagents for parallel purification. J. Am. Chem. Soc., Washington, v. 119, n. 21, p. 4882, 1997.

BORMAN, S. New alternative to bead synthesis. Chem. Eng. News, Washington, v. 79, n. 23, p. 49-50, 2001.

BORMAN, S. Combinatorial chemistry. Chem. Eng. News, Washington, v. 79, n. 35, p. 49, 2001.

BROCCHINI, S. Combinatorial chemistry and biomedical polymer development. Adv. Drug Deliv. Rev., Amsterdam, v. 53, p. 123-130, 2001.

BUNIN, B. A.; ELLMAN, J. A. A general and expedient method for the solid-phase synthesis of 1,4benzodiazepine derivatives. J. Am. Chem. Soc., Michigan, v. 114, n. 27, p. 10997-10998, 1992.

CALDWELL, J. W. Patents in Combi-Space: patent challenges in combinatorial chemistry. Biotechnol. Bioeng., New York, v. 61, n. 1, p. 69-75, 1998.

CHAPLEUR, Y.; MOITESSIER, N.; DUFOUR, S.; CHRÉTIEN, F.; THIERY, J. P.; MAIGRET, B. Design, synthesis and preliminary biological evaluation of a focused combinatorial library of stereodiverse carbohydrate-scaffold-based peptidomimetics. Bioog. Med. Chem. Lett., Oxford, v. 9, p. 511-523, 2001.

CURRAN, D. P.; HADIDA, S.; STUDER, A.; HE, M.; KIM, S. Y.; LUO, Z.; LARHED, M.; HALLBERG; LINCLAU, B. Experimental techniques in fluorous synthesis: a user's guide. In: FENNIRI, H., ed. Combinatorial chemistry. Practical approach. Oxford: Oxford University Press, 2000. cap. 11, p. 327-352.

De MIGUEL, Y. R.; SHEARER, A. S. Infrared spectroscopy in solid-phase synthesis. Biotechnol. Bioeng., New York, v. 71, n. 2, p. 119-129, 2000/2001. 
DE WITT, S. H.; KIELY, J. S.; STANKOVIC, C. J.; SCHROEDER, M. C.; CODY, D. M. R.; PAIVA, M. R.; 'Diversomers': An approach to nonpeptide, nonoligomeric chemical diversity. Proc. Nat. Acad. Sci. USA, Washington, v. 90, n. 15, p. 6909-6913, 1993.

DIAS, R. L. A.; CORREA, A. G. Aplicações da química combinatória no desenvolvimento de fármacos. Quim. Nova, São Paulo, v. 24, n.2, p. 236-242, 2001.

DULAC, C.; FRASER, S.E.; STARLING, B. New technologies. Curr. Op. Neurobiol., London, v. 11, p. 591-592, 2001.

EDWARDS, P. Combinatorial chemistry. Azole antifungals. Drug Discov. Today, v. 6, n. 18, p. 965, 2001.

EIFLER-LIMA, V.L. ICS-UNIDO Workshop on Trends and Applications of Combinatorial Chemistry and Combinatorial Technologies. São Pedro/SP. 2002.

FAUCHÉRE, J-L.; BOUTIN, J.A.; HENLIN, J-M.; KUCHARCZYK, N.; ORTUNO, J-C. Combinatorial chemistry for the generation of molecular diversity and the discovery of bioative leads. Chemom. Intel. Lab. Syst., Barcelona, v. 43, p. 43-68, 1998.

FENNIRI, H. Combinatorial Chemistry. Oxford: Oxford Press, 2000. $476 \mathrm{p}$.

FOLKERS, G.; KUBINYI, H. Vers une conception rationnelle des médicaments. Pour la Science, Paris, n. 241, p. 40-47, 1997.

FRANZÉN, R. G. Recent advances in the preparation of heterocycles on solid support: A review of the literature. J. Comb.Chem., Washington, v.2, n.3, p. 195-214, 2000.

GANESAN, A. Recent developments in combinatorial organic synthesis. Drug Discov. Today, Toronto, v. 7, n. 1, p. 47-55, 2002.

GAYO, L. M. Solution-phase library generation: Methods and applications in drug discovery. Biotechnol. Bioeng., New York, v .61, n. 2, p. 95-106, 1998.

GEDECK, P.; WILLETT, P. Visual and computacional analysis of structure-actiivity relationships in highthroughput screening data. Curr. Op. Chem Biol., London, v. 5, p. 389-395, 2001.
GORDON, E. M.; KERWIN Jr, J. F. Combinatorial chemistry and molecular diversity in drug discovery. New York: Wiley-Liss, 1998. 516 p.

GRAVERT, D.; JANDA, K. D. Organic synthesis on soluble polymer supports: liquid-phase methodologies. Chem. Rev., Washington, v. 97, p. 489, 1996.

HANS, H. S.; WOLFE, M. M.; BRENNER, S.; JANDA, K. D. Liquid-phase combinatorial synthesis. Proc. Nat. Acad. Sci. USA, Washington, v. 92, n. 14, p. 6419-6423, 1995 .

HUESGO, J. A. Workshop "Novas Aproximações para a Descoberta de Fármacos. Possibilidades e Limitações na América Latina" CYTED, 19 a 24 de agosto de 2001. Rio de Janeiro/Brasil.

KUBINYI, H. Strategies and recent technologies in drug discovery. Pharmazie, Berlin, v. 50, n. 10, p. 647-662. 1995.

MARQUARDT, M. M.; EIFLER-LIMA, V. L. Química Combionatória. Em busca da diversidade. In: ANDREI, C. C. FERREIRA, D. T., FACCIONE, M., FARIA, T. J., eds. Da Química medicinal à Química combinatória e modelagem molecular - Um curso prático. Barueri: Manole, 2002. 154 p.

MARQUARDT, M. M.; EIFLER-LIMA, V. L. A síntese orgânica e seus suportes poliméricos mais empregados. Quím. Nova, São Paulo, v. 24, n. 6, p.846-855, 2001.

MCLEAN, D.; BALDWIN, J. J.; IVANOV, V. T.; KATO, Y.; SHAW, A.; SCHNEIDER, P.; GORDON, M. Glossary of therms used in combinatorial chemistry. J. Comb. Chem., Washington, v. 2, p. 562-78, 2000.

MERRIFIELD, R. B. Solid phase peptide synthesis. I. The synthesis of a tetrapeptide. J. Am. Chem. Soc., New York, v. 85, p. $2149,1963$.

PIVONKA, D. E. On-bead quantitation of resin bound functional groups using analogue techniques with vibrational spectroscopy. J. Comb. Chem., Washington, v. 2, p. 33-38, 2000 .

PROKAI, L. Combinatorial lead optimization of a neuropeptide FF antagonist. J. Med. Chem., New York, v. 44, p. 1623-1626, 2001. 
RANO, T. A.; CHENG, Y.; HUENING, T. T.; ZHANG, F.; SCHLEIF, W. A.; GABRYELSKI, L.; OLSEN, D. B.; KUO, L. C.; JIUNN, H. L.; XU, X.; OLAH, T. V.; MCLOUGHLIN, D. A.; KING, R.; CHAPMAN, K. T.; TATA, J. R. Combinatorial diversification of indinavir: In vivo mixture dosing of an HIV protease inhibitor library. Bioog. Med. Chem. Lett., Oxford, v. 10, p. 1527-11530, 2000.

SHERRINGTON, D. C. Preparation, structure and morphology of polymers supports. Chem. Commun., London, v. 21, p. 2275-2286, 1998.

SIGAL, N. H.; CHELSKY, D. Approaches and technologies for screening large combinatorial libraries. In: GORDON, E. M.; KERWIN JR., J. F., ed., Combinatorial chemistry and molecular diversity in drug discovery. New York: Wiley-Liss, 1998.

SRINIVASA, N.T.; GUPTA, P.; KUNDU, B. Solid-phase synthesis of arylalkanolamines. Tetrahedron Lett., Oxford, v. 42, n. 34, p. 5993-5995, 2001.

STUDER, A.; HADIDA, S.; FERRITO, R.; KIM, S.; JEGER, P.; WIPF, P.; CURRAN, D. P. A strategic alternative to solid phase synthesis: preparation of a small isoxazoline library by "fluorous synthesis". Tetrahedron, Oxford, v. 53, n. 19, p. 6681-6696, 1997.

TERRETT, N. Combinatorial chemistry - Synthesis of lactams. Drug Discov. Today, Amsterdam, v. 4, n. 12, p. 570-572, 1999.

THOMPSON, L. A.; ELLMAN, J. A. synthesis and applications of small molecule libraries. Chem. Rev., Washington, v. 96, p. 555-600, 1996.

TRIAS, J. The role of combichem in antibiotic discovery. Curr. Op. Microbiol., London, v. 4, p. 520-525, 2001.
VILLAR, H.O.; KOEHLER, R.T. Comments on the design of chemical libraries for screening. Mol. Diversity, Arizona, v. 5, n. 1, p. 13-24, 2000.

WATKINS, K. J. Fighting the clock. Chem. Eng. News, Washington, p. 27-34, 2002.

WELLS, W. Combinatorial Chemistry- making more drugs,

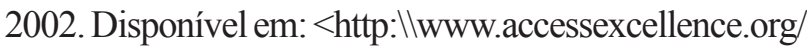
AB/BA/combiChem/>. Acesso em: 04 de fev. 2004.

WERMUTH, C. G. ed. The practice of medicinal Chemistry. London: Academic Press, 1996. 968 p.

WOLCKE, J.; ULLMANN, D. Miniaturized HTS technologies - uHTS. Drug Discov. Today, Amsterdam, v. 6, n. 12 , p. 637-646, 2001.

YAN, B. Monitoring the progress and the yield of solid-phase organic reactions directly on resin supports. Acc. Chem. Res., Washington, v. 31, p. 621-630, 1998.

YAN, B.; GREMLICH, H.U.; MOSS, S.; COPPOLA, G. M.; SUN, Q.; LIU, L. A Comparison of various FTIR and FT Raman methods: applications in the reaction optimization stage of combinatorial chemistry. J. Comb. Chem., Washington, v. 1, n. 1, p. 46-54, 1999.

YUNES, R.A.; CECHINEL FILHO, V. Breve análise histórica da química de plantas medicinais: Sua importância na atual concepção de fármaco segundo os paradigmas ocidental e oriental. In: YUNES, R. A.; CALIXTO, J.B., eds. Plantas medicinais. Sob a ótica da química medicinal moderna. Chapecó: Editora Argus, 2001. p. 19-46.

Recebido para publicação em 13 de agosto de 2002. 